\title{
Prevention and management of vision loss relating to facial filler injections
}

Kwok Thye David Loh ${ }^{1}$, MBBS, Jun Jin $\underline{\text { Chua }}{ }^{2}$, MBBS, FRCS, Hung Ming Lee $\underline{\text { I }}^{3}$, MBBS, FRCS, Joyce Teng-Ee $\underline{\operatorname{Lim}}^{4}$, FRCP, FAMS, Gerard $\underline{\text { Chuah }}^{5}$, MBBS, FRCSEd, Benjamin $\underline{\text { Yim}}^{6}$, MBBS, MSc, Boon Kwang $\underline{\text { Puah }}^{7}$, MBBS, GDFP

INTRODUCTION With the increased use of filler and fat injections for aesthetic purposes, there has been a corresponding increase in the incidence of complications. Vision loss as an uncommon but devastating vascular side effect of filler injections was the focus of this paper.

METHODS A review committee, consisting of plastic surgeons, aesthetic medical practitioners, ophthalmologists and dermatologists from Singapore, was convened by the Society of Aesthetic Medicine (Singapore) to review and recommend methods for the prevention and management of vision loss secondary to filler injections.

RESULTS The committee agreed that prevention through proper understanding of facial anatomy and good injection techniques was of foremost importance. The committee acknowledged that there is currently no standard management for these cases. Based on existing knowledge, injectors may follow a proposed course of action, which can be divided into immediate, definitive and supportive. The goals were to reduce intraocular pressure, dislodge the embolus to a more peripheral location, remove or reverse central ischaemia, preserve residual retinal function, and prevent the deterioration of vision. Dissolving a hyaluronic acid embolus remains a controversial option. It is proposed that injectors must be trained to recognise symptoms, institute immediate actions and refer patients without delay to dedicated specialists for definitive and supportive management.

CONCLUSIONS Steps to prevent and manage vision loss based on current evidence and best clinical practices are outlined in this paper. Empirical referral to any emergency department or untrained doctors may lead to inordinate delays and poor outcomes for the affected eye.

Keywords: aesthetic medicine, blindness, facial fillers, filler injection, hyaluronic acid

\section{INTRODUCTION}

Soft-tissue augmentation of the face for aesthetic purposes has become increasingly popular in Singapore. Materials that are frequently used include autologous fat, hyaluronic acid gel, calcium hydroxyapatite and polylactic acid, among others. These procedures are generally safe; their complications, such as erythema or ecchymosis, are generally mild and often transient. ${ }^{(1)}$ Very rarely, severe complications such as skin necrosis, visual loss and stroke have been reported.(2) It is believed that these events are caused by accidental intravascular administration of filler material leading to ischaemia or infarction distal to the point of entry.

Blindness following filler injections was the focus of this paper. The exact incidence of this devastating adverse event remains to be determined due to the heterogeneity of data. Most cases in Asia are documented in South Korea, where filler injections are common. ${ }^{(3)}$ However, case reports have also emerged from China. While injections into other facial sites (e.g. scalp, cheek, lips, temple, frontal area, etc) have been documented to cause vascular complications, ${ }^{(4,5)}$ injections into the nose and the glabella form the vast majority of reported cases of blindness. This is of particular significance in Singapore, where nose enhancement is a commonly requested procedure by the majority Oriental population. ${ }^{(6)}$
As the face ages, the skin loses its elasticity and decreases in thickness, fat pads atrophy, and the facial bones begin to resorb. These anatomical changes result in ptosis. Today, facial fillers $^{(7)}$ are commonly used for augmentation to provide support to the ageing face or anteriorly project facial features such as the forehead, nasal bridge and chin. It is important to note that facial vasculature varies. Patients who have previously undergone rhinoplasty or facial reconstruction, for instance, may have an unusual distribution of facial vasculature due to neovascularisation and tissue reorganisation. The anatomy discussed in this paper relates to the majority of the general population. ${ }^{(8)}$

Branches of the angular artery supply the inferomedial aspect of the orbital fossa and the dorsum of the nose (Fig. 1a). Terminal branches of the ophthalmic artery, namely the supraorbital and supratrochlear arteries, supply the medial forehead (Fig. 1b). Anastomoses between the terminal branches of the angular artery, and the supratrochlear and supraorbital arteries are well documented. Hence, accidental intravascular injections of filler material into the dorsal nasal artery at sufficient pressure can push a column of material into the supratrochlear or supraorbital arteries. This column may travel retrograde to a point proximal to the artery's origin at the ophthalmic artery. Following systolic pressure, material may be dislodged from the column and propelled distally along the ophthalmic artery itself or into

${ }^{1}$ David Loh Surgery, ${ }^{2} \mathrm{JJ}$ Chua Rejuvenative Cosmetic \& Laser Surgery, ${ }^{3}$ Lee Hung Ming Eye Centre, Gleneagles Hospital, ${ }^{4}$ Joyce Lim Skin and Laser Clinic, ${ }^{5}$ Total Eyecare Center, ${ }^{6}$ Dr Benjamin Yim Clinical Aesthetics \& Laser Centre, ${ }^{7}$ Amaris B Clinic, Singapore

Correspondence: Dr Loh Kwok Thye David, General Practitioner, David Loh Surgery, 501 Orchard Road, \#05-16 Wheelock Place, Singapore 238880. dr@davidloh.sg 

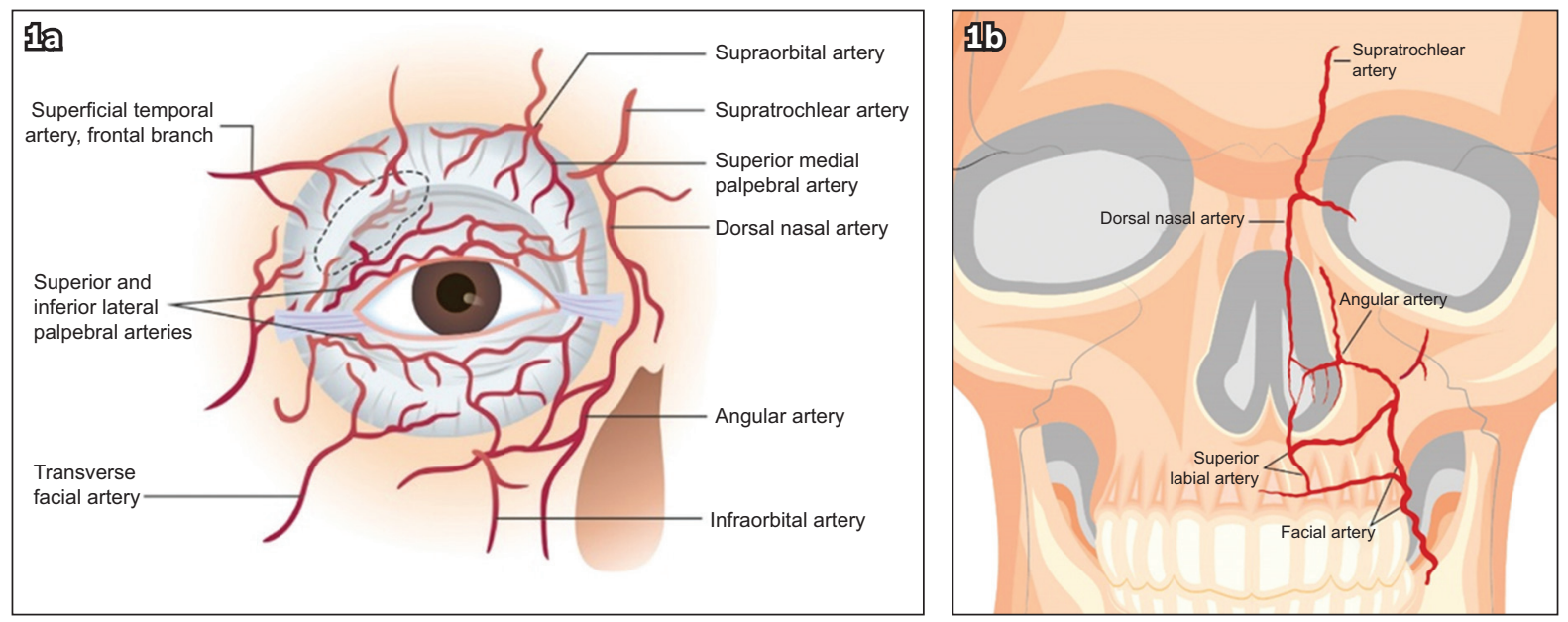

Fig. 1 Diagrams show a schematic view of the blood supply to the midface with (a) eyelid vasculature and (b) vasculature of the nasolabial area. ${ }^{(10)}$

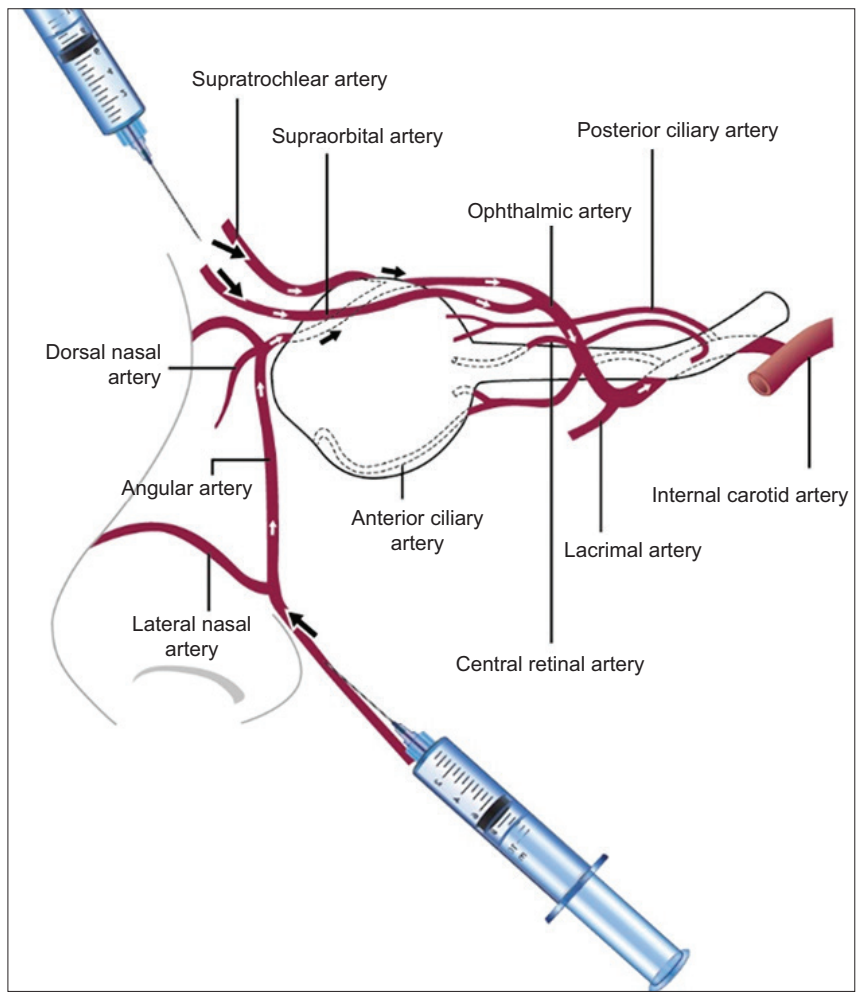

Fig. 2 Diagram shows a schematic view of the injection sites of possible anterograde or retrograde flow of fillers. ${ }^{(7)}$

the central retinal artery, causing sudden vision loss (Fig. 2). ${ }^{(9)}$ Blindness has been reported with the use of both blunt cannulas and sharp needles, as well as small- and large-bore cannulas. ${ }^{(9)}$

The main argument in favour of using cannulas is that they are less likely to puncture a blood vessel. Proponents of using sharp needles argue that a sharp needle that is introduced perpendicular to the skin will easily penetrate both walls of the arteries, whereas a fine cannula, introduced tangentially along the direction of the course of the artery, remains within the lumen if it punctures the artery. If the artery is cannulated and a filler injected, a column of filler material will likely build

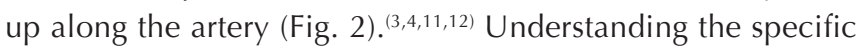
anatomical planes at the area of injection is also important. For the nose, the supraperiosteal or supraperichondrial planes are relatively bloodless. After exiting from the respective foramina or notch, the supratrochlear and supraorbital arteries run in a plane superficial to or within the frontalis muscle. Hence, injections via needle or cannula should be applied deep into these areas. ${ }^{(11)}$

Blindness resulting from filler embolisation in the eye occurs within seconds after injection, regardless of ocular pain in the affected eye. Pain at the injection site is a possibility. ${ }^{(3-6,9,11,12)}$ Other manifestations of this kind of occlusion include ophthalmoplegia, horizontal strabismus, ptosis, corneal oedema, skin necrosis and phthisis bulbi. Ophthalmic artery occlusion is characterised by severe ocular pain minutes after the injection. On the other hand, central retinal and branch artery occlusions may present with vision loss but without ocular pain. ${ }^{(9)}$

In theory, lowering intraocular pressure may dislodge the embolus into more peripheral retinal vessels, resulting in increased perfusion and preventing further damage to still-viable central retinal tissue. ${ }^{(4)}$ To lower intraocular pressure, topical instillation of timolol, and/or oral acetazolamide and ocular massage can be performed. These measures can be commenced by a non-ophthalmologist injector while arrangements are being made to transfer the patient. ${ }^{(4)}$ In addition, anterior chamber paracentesis performed by an ophthalmologist can further decrease intraocular pressure. ${ }^{(3,4,9)}$

Hyaluronic acid is commonly used as filler in Singapore. Theoretically, hyaluronidase can be used to break down the hyaluronic acid filler material inadvertently injected into the vessel in the hope of reversing ischaemia. This enzyme can be injected into the retrobulbar space using a cannula or directly into the incriminating vessel, although results of the limited cases reportedly treated with these two methods have been equivocal. . $3,4,11,13,14)$ It is postulated that this enzyme may diffuse across the wall of the ophthalmic artery. However, this method is not useful for central retinal artery events. It has been suggested that if hyaluronidase is injected into the supraorbital or supratrochlear artery, it will also reach the filler material inside the ophthalmic artery, similar to the route taken by the filler embolus. Attempts to dissolve the filler material should only be performed by an experienced ophthalmologist. There is a 60-90 minute 
window to either dislodge or dissolve the filler material within the artery before irreparable visual loss. ${ }^{(11)}$ Supportive care involves the use of hyperbaric oxygen and anticoagulation, among other measures, which are intended to recover any salvageable retinal tissue.

\section{METHODS}

A review committee consisting of aesthetic medical practitioners, plastic surgeons, ophthalmologists and dermatologists from Singapore was convened by the Society of Aesthetic Medicine (Singapore) on 24 March 2015. The objectives of this committee were to review evidence on methods of prevention and management of vision loss secondary to filler injections, and develop a reference protocol for use in local centres for practitioners who may encounter these events.

A literature search was conducted for case reports and case series of dermal filler complications from January 2000 to March 2015. The search terms 'dermal filler', 'facial filler', 'filler', 'blindness', 'vision loss' and 'complications' were keyed into the search engines PubMed MEDLINE ${ }^{\circledR}$, ClinicalKey ${ }^{\circledR}$ and Google Scholar. Articles that recommended preventive and secondary management of vision loss were reviewed by the committee and rated according to applicability and level of evidence (Table I). A total of six case series and seven isolated case reports on vision loss were finally included in the review. Only articles that discussed vision loss, permanent or transient, were considered for further analysis, regardless of the presence of other associated symptoms and type of filler used. There is limited data in the literature on the incidence of blindness resulting from filler embolisation, and there are no published cases in Singapore to the best of our knowledge. All published studies were retrospective in nature and it is unclear how many cases were unreported from point of contact, as the cases were referrals to retinal specialty centres.

Following a discussion on the anatomy and relevant structures of the midface, the committee arrived at a set of recommendations that were further evaluated following a grading system based on the 2011 Oxford Centre for Evidence-Based Medicine's Grades of Recommendation ${ }^{(15)}$ (Table I).

\section{RESULTS AND DISCUSSION}

The committee divided the recommendations into four main sets. The first category, 'pre-injection', includes recommendations on understanding anatomy, injection technique and choice of needle versus cannula. The other sets pertain to 'immediate', 'definitive' and 'supportive' management. ${ }^{(12,16)}$ As hyaluronic acid-based fillers are more common in Singapore, measures for managing this compound are more extensively discussed.

\section{Pre-injection}

- $\quad$ Pre-injection recommendations are intended to prevent complications. Having a thorough knowledge of anatomy and injection techniques is perhaps the most important of all the recommendations. (Grade A)

- Although embolism can develop from any of the injected
Table I. Grades of recommendation based on the Oxford Centre for Evidence-Based Medicine (CEBM) grading system. ${ }^{(15)}$

\begin{tabular}{|c|c|c|}
\hline $\begin{array}{l}\text { Grade of } \\
\text { recommendation }\end{array}$ & $\begin{array}{l}\text { Level of } \\
\text { evidence }\end{array}$ & Type of study \\
\hline \multirow[t]{2}{*}{ A } & $1 \mathrm{a}$ & $\begin{array}{l}\text { Systematic review of (homogeneous) } \\
\text { randomised controlled trials (RCTs) }\end{array}$ \\
\hline & $1 b$ & $\begin{array}{l}\text { Individual RCTs (with narrow } \\
\text { confidence intervals) }\end{array}$ \\
\hline \multirow[t]{4}{*}{ B } & $2 a$ & $\begin{array}{l}\text { Systematic review of (homogeneous) } \\
\text { cohort studies }\end{array}$ \\
\hline & $2 b$ & $\begin{array}{l}\text { Individual cohort study or } \\
\text { low-quality RCTs }\end{array}$ \\
\hline & $3 a$ & $\begin{array}{l}\text { Systematic review of (homogeneous) } \\
\text { case-control studies }\end{array}$ \\
\hline & $3 b$ & Individual case-control studies \\
\hline C & 4 & $\begin{array}{l}\text { Case series, or low-quality cohort or } \\
\text { case-control studies }\end{array}$ \\
\hline $\mathrm{D}$ & 5 & $\begin{array}{l}\text { Expert opinions based on } \\
\text { non-systematic reviews of results or } \\
\text { mechanistic studies }\end{array}$ \\
\hline
\end{tabular}

These ratings can be found in parentheses beside key recommendations in this article. Recommendations that are not fully applicable to facial filler complications but have been evaluated for thromboembolism caused by other aetiologies are classified as CEBM Grade D.

facial areas, the glabella and nose are most at risk for puncture of the blood vessels. ${ }^{(3-6,9,11)}$ It is therefore advised that utmost caution be applied when treating these areas. (Grade C)

- $\quad$ Approved filler substances appear to be generally safe; however, a small risk of adverse effects has been identified with all of these substances. Among these, autologous fat has been shown to be associated with higher risk of vascular adverse events (e.g. higher diffuse occlusion rate, a worse visual prognosis and a greater incidence of combined cerebral infarction) than other fillers. ${ }^{(3-6,9,11)}$ (Grade B)

- The use of a standard pre-injection counselling sheet is encouraged to state the potential adverse effects from any filler and symptoms to watch out for, and outline postprocedural care instructions. ${ }^{(16)}$ (Grade D)

\section{Injection technique}

- Using small-bore needles and cannulas is the standard recommendation for administering filler materials, as they are potentially less traumatic. ${ }^{(4)}$ The use of blunt cannulas in high-risk regions is thought to decrease the chance of penetrating a vessel wall compared to conventional bevel-edged sharp needles. ${ }^{(4,9,12)}$ However, if a vessel wall is breached, there is a high chance that the blunt cannula will remain within the vessel and introduce a column of filler within the vessel. (Grade D)

- If a sharp needle is being used, a perpendicular injection directly in contact with the bone is recommended; injecting into a deeper plane may avoid the vessels. (Grade D)

- $\quad$ Aspiration to observe for backflow of blood may be useful to detect accidental intravascular placement of the cannula or needle tip. (Grade D) 


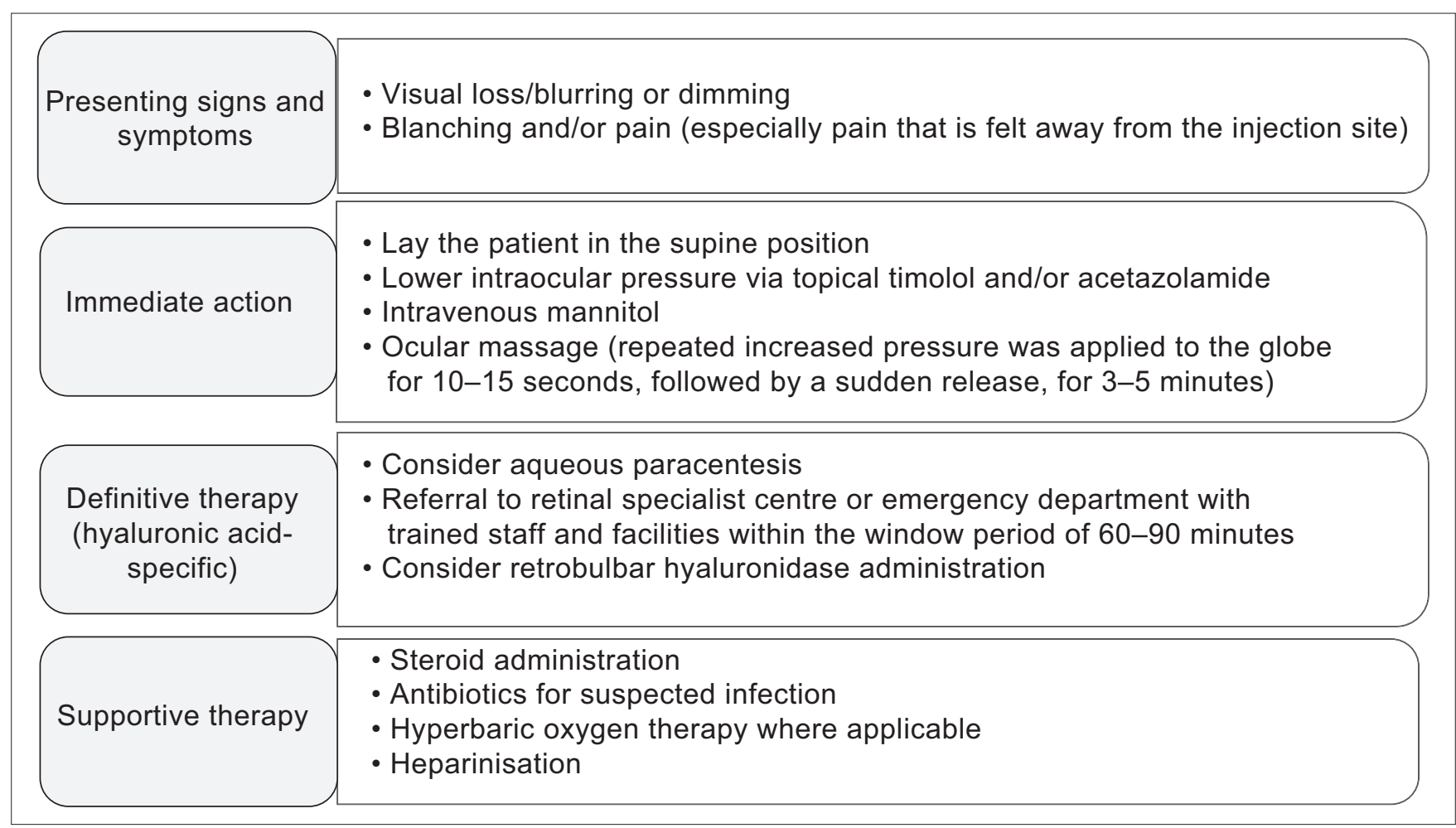

Fig. 3 Treatment algorithm for addressing vision loss following filler injections.

- Injections should be performed slowly (i.e. not bolus), with as little pressure on the plunger as possible while watching out for symptoms of pain and signs of blanching or mottling of the skin that is supplied by the vessel. In the event of intravascular injection, this might prevent filler material from being propelled in a retrograde fashion into the artery. ${ }^{(4)}$ (Grade C)

\section{Compression on the side of the nasal bridge to prevent propagation of thrombosis or embolus}

Another suggested technique to prevent embolism of filler material is digital compression of the inferior-medial orbital rim and the sides of the nose. ${ }^{(17)}$ (Grade D) More research is required to determine the effectiveness of this method, beyond cadaveric studies, in obstructing emboli progression within anastomosing vessels.

\section{Management of sudden visual loss during filler treatments}

The committee reached an agreement on the following points for recommendation based on best practices and the recommendations stated in the reviewed case reports and series mentioned in the Methods section (Fig. 3).

\section{Presenting signs and symptoms}

- Vascular occlusion can manifest as blanching followed by mottling discolouration (livedo reticularis) over the area of occlusion. ${ }^{(12)}$ Other symptoms outlined in the literature include toothache and headache. (Grade C)

- When pain is felt in a location away from the injection area, it is highly probable that an embolism has occurred, as it indicates intravascular movement of material. Vision loss secondary to filler embolisation may be accompanied by pain in the affected eye. (Grade D)

- Upon suspicion of vascular compromise to the retina, injection should be stopped and the following steps considered. To optimally treat these cases, these measures should be taken within the initial 60-90 minutes from symptom onset. The study by the European Assessment Group for Lysis in the Eye found that in patients suspected of having thromboembolism, those with a shorter duration of symptoms had a better visual prognosis. ${ }^{(4)}$ (Grade D)

\section{Immediate action}

These recommendations are intended to quickly lower intraocular pressure and dislodge the embolus to a more peripheral downstream location. ${ }^{(4)}$ If vascular compromise to the retina is suspected, the patient should be placed in a supine position and the following should be immediately administered: (a) one drop of topical timolol $0.5 \%$ and/or an acetazolamide $500 \mathrm{mg}$ tablet (after excluding for sulfa allergy) ${ }^{(1,4,9)}$ (Grade B); (b) an intravenous infusion of mannitol 20\% $\%^{(1,9,11)}(100 \mathrm{~mL}$ over 30 minutes); and (c) nitroglycerin $2 \%$ paste. ${ }^{(5,13,14,18)}$ (Grade D) Rapid infusion of mannitol may precipitate shrinkage of the cerebrospinal fluid space and stretching of the transdural veins, leading to slow cerebral haemorrhage in some patients. It is best to avoid this agent in the elderly and those with a past history of stroke or on blood thinning agents.

Once the drugs have been administered, ocular massage should commence (Fig. 4). ${ }^{(3-6,9,12)}$ Massage lowers intraocular pressure and increases blood flow in the arterioles, potentially dislodging the embolus; however, there are currently mixed results in a number of case series. ${ }^{(1,4)}$ (Grade C) The committee recommends the following 


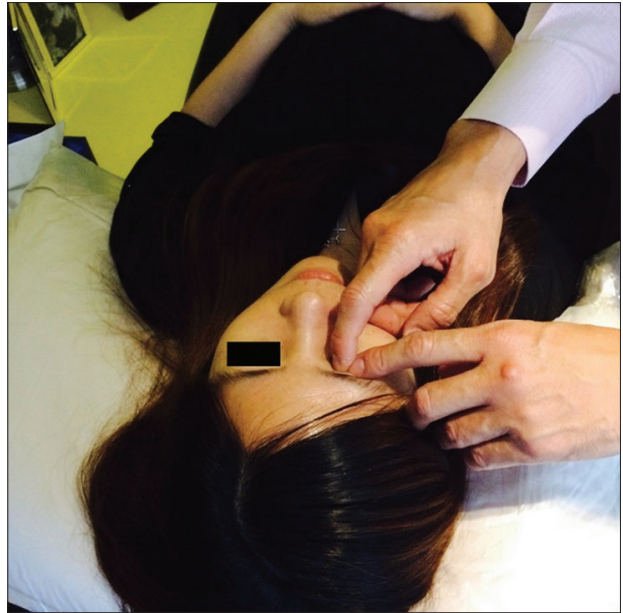

Fig. 4 Photograph shows the correct technique for ocular massage.

simple digital massage for non-ophthalmologists: (a) Ensure that the patient's eyes are closed. (b) Apply firm pressure on the eyeball through the closed eyelids. Pressure should be firm enough that the eyeball is indented about 2-3 mm. (c) Apply firm pressure for 5-15 seconds and quickly release. (d) Repeat this cycle for at least five minutes. (e) At the end of five minutes, check if the patient's vision in the affected eye is brighter.

Most ophthalmologists perform ocular massage using a Goldmann fundus contact lens, following these steps: (a) Apply topical anaesthetic on the affected eye. (b) Place the Goldman fundus contact lens directly on the corneal surface with some lubricant eye ointment (e.g. carbomer-based ophthalmic lubricants). (c) Apply direct firm pressure (i.e. the eyeball is indented about 2-3 mm). Apply firm pressure on the Goldman fundus contact lens for about 5-15 seconds and quickly release. The ophthalmologist can directly visualise the retinal vessels using the fundus contact lens and check if the embolus has been dislodged. (d) The cycle of applying firm pressure for 5-15 seconds followed by quick release should be repeated for at least five minutes, or until the ophthalmologist can confirm that the embolus has been dislodged.

It is important to note that ocular massage is not currently supported by high-quality evidence for treating Central Retinal Artery Occlusion, with few successful case reports published. In addition, the method of ocular massage may vary greatly between ophthalmologists and requires evaluation in larger studies. Other reports in the literature suggest that the use of acetylsalicylic acid is useful for other emboli, but its efficacy and that of other antiaggregants has not been proven for filler-related retinal vascular complications. ${ }^{(12)}$ (Grade D)

\section{Definitive therapy}

- Aqueous paracentesis was documented to be useful for rapidly decreasing intraocular pressure. ${ }^{(4,9)}$ As this procedure requires highly specialised skill and equipment, it should only be performed by a trained ophthalmologist. (Grade D)

- Hyaluronic acid is a naturally occurring, biodegradable glycosaminoglycan. Due to its large size relative to some arterioles (i.e. 20-1000 $\mu \mathrm{m}$ ), it may become lodged, causing distal tissue necrosis. ${ }^{(2)}$ If hyaluronic acid embolism occurs, there is a chance that it can be dissolved by hyaluronidase administration within the window period of 60-90 minutes. $^{(11)}$ (Grade D)

- $\quad$ The preferred method of administration of hyaluronidase remains debatable, along with the safety of these methods. It has been suggested that retrobulbar injection of hyaluronidase may be able to dissolve hyaluronic acid; 2-4 mL of hyaluronidase (150-200 units $/ \mathrm{mL}$ ) can be injected into the inferolateral retrobulbar space with a cannula. ${ }^{(11)}$ (Grade D) Another suggested method involves dissecting the eyelid near the brow to locate the supraorbital artery and inject hyaluronidase retrograde into the ophthalmic artery. Yet another suggested technique is to directly inject the enzyme into the ophthalmic artery following neuroradiologic imaging. These procedures are highly skill-dependent and only theoretically beneficial at this point in time. ${ }^{(17)}$ (Grade D) - $\quad$ Some believe that an attempt should be made to dissolve a hyaluronic acid filler embolus to the eye. If the ophthalmologist is comfortable with the procedure, the potential benefit of averting catastrophic blindness is more critical than the risk of injury to the eye. (Grade D)

\section{Supportive therapy}

Supportive therapy is recommended after the aforementioned acute measures in order to help heal and salvage any viable retinal cells. A few studies have documented some recovery of residual vision after administration of these measures.

- $\quad$ Steroid administration was performed in a few cases, with variable results. ${ }^{(4)}$ (Grade D)

- Judicious use of topical or intravenous antibiotics for suspected infection is acceptable. ${ }^{(4,16)}$ (Grade D)

- Hyperbaric oxygen therapy may reverse any salvageable retinal damage. ${ }^{(4,16)}$ (Grade D)

- Lastly, anticoagulation by heparinisation may also be considered to prevent further thrombosis. ${ }^{(4)}$ (Grade D)

In conclusion, vascular occlusions are uncommon complications associated with facial soft-tissue augmentation with fillers or autologous fat. Vision loss is a subset of these vascular complications that is of particular interest, as it causes a severe form of complication brought about by an elective procedure. This paper identified several key recommendations to prevent and treat blindness based on available recommendations in literature and best practices from experts in Singapore.

Prevention through a good understanding of facial vasculature anatomy and injection techniques is important. Both sharp needles and blunt cannulas are safe in experienced hands. In the event of vision loss relating to filler injections, there are immediate actions that can be taken by the injector and ophthalmologist to decrease intraocular pressure and hopefully dislodge the embolus peripherally. No universal consensus exists on the best way to dissolve and dislodge a hyaluronic acid embolus, but the committee opines that such measures may be attempted to save the eye, despite a small risk of further injury to the eye, 
only because the alternative to attempting to dissolve the filler is blindness. The supportive measures that have been discussed are also well documented in the literature for non-filler-related thromboembolism, and may thus be considered for filler-related cases. In order to minimise stress to the patient and ensure the best outcome, it is recommended that the centres that are equipped to receive such patients be identified and channels of direct communication established in the future.

\section{REFERENCES}

1. Hsiao SF, Huang YH. Partial vision recovery after iatrogenic retinal artery occlusion. BMC Ophthalmol 2014; 14:120.

2. Tansatit T, Moon HJ, Apinuntrum P, Phetudom T. Verification of Embolic Channel Causing Blindness Following Filler Injection. Aesthetic Plast Surg 2015; 39:154-61.

3. Park KH, Kim YK, Woo SJ, et al. latrogenic occlusion of the ophthalmic artery after cosmetic facial filler injections: a national survey by the Korean Retina Society. JAMA Ophthalmol 2014; 132:714-23.

4. Lazzeri D, Agostini T, Figus M, et al. Blindness following cosmetic injections of the face. Plast Reconstr Surg 2012; 129:995-1012.

5. Chen Y, Wang W, Li J, et al. Fundus artery occlusion caused by cosmetic facial injections. Chin Med J (Engl) 2014; 127:1434-7.

6. Sundaram H, Carruthers J. Glabella/central brow. 2013. In: Soft Tissue Augmentation, 3rd edition: Procedures in Cosmetic Dermatology Series (Expert Consult - Online and Print) [online]. Available at: http://clinicalgate. com/glabellacentral-brow/. Accessed March 21, 2015.

7. Ballin AC, Brandt FS, Cazzaniga A. Dermal fillers: an update. Am J Clin Dermatol 2015; 16:271-83.

8. De Boulle K, Heydenrych I. Patient factors influencing dermal filler complications: prevention, assessment, and treatment. Clin Cosmet Investig Dermatol 2015; 8:205-14.
9. Park SW, Woo SJ, Park $\mathrm{KH}$, et al. Iatrogenic retinal artery occlusion caused by cosmetic facial filler injections. Am J Ophthalmol 2012; 154:653-662.e1.

10. Inoue K, Sato K, Matsumoto D, Gonda K, Yoshimura K. Arterial embolization and skin necrosis of the nasal ala following injection of dermal fillers. Plast Reconstr Surg 2008; 121:127e-128e.

11. Carruthers JD, Fagien S, Rohrich RJ, Weinkle S, Carruthers A. Blindness caused by cosmetic filler injection: a review of cause and therapy. Plast Reconstr Surg 2014; 134:1197-201.

12. Kim JH, Ahn DK, Jeong HS, Suh IS. Treatment algorithm of complications after filler injection: based on wound healing process. J Korean Med Sci 2014; 29 Suppl 3:S176-82.

13. Choi WY, Cho HW, Lee DW. Complications of Injectable Soft Tissue Filler. Arch Aesthetic Plast Surg 2015; 21:1-6.

14. Oh BL, Jung C, Park KH, Hong YJ, Woo SJ. Therapeutic intra-arterial hyaluronidase infusion for ophthalmic artery occlusion following cosmetic facial filler (hyaluronic acid) injection. Neuroophthalmol 2014; 38:39-43.

15. OCEBM Levels of Evidence Working Group. Oxford Centre for Evidencebased Medicine - Levels of Evidence (March 2009) [online]. Available at: http://www.cebm.net/oxford-centre-evidence-based-medicine-levelsevidence-march-2009. Accessed March 21, 2015.

16. Kim DW, Yoon ES, Ji YH, et al. Vascular complications of hyaluronic acid fillers and the role of hyaluronidase in management. J Plast Reconstr Aesthet Surg 2011; 64:1590-5.

17. Tansatit T, Apinuntrum $P$, Phetudom T. A cadaveric feasibility study of the intraorbital cannula injections of hyaluronidase for initial salvation of the ophthalmic artery occlusion. Aesthetic Plast Surg 2015; 39:252-61.

18. Liao J, Ehrlich M, Woodward JA. Soft Tissue Fillers: Avoiding and Treating Complications. In: American Academy of Opthalmology EyeNet ${ }^{\circledR}$ Magazine [online]. Available at: http://www.aao.org/eyenet/article/softtissue-fillers-avoiding-treating-complication?february-2013. Accessed March 17, 2015. 